\title{
Discrete natural neighbour interpolation with uncertainty using cross-validation error-distance fields
}

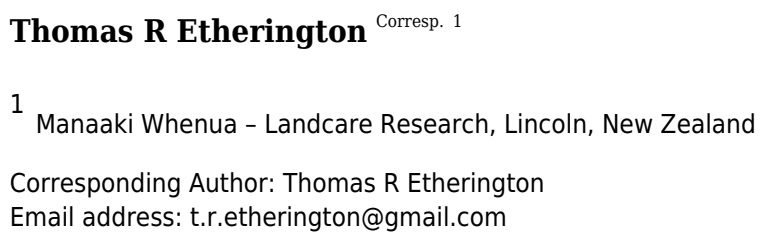

Interpolation techniques provide a method to convert point data of a geographic phenomenon into a continuous field estimate of that phenomenon, and have become a fundamental geocomputational technique of spatial and geographical analysts. Natural neighbour interpolation is one method of interpolation that has several useful properties: it is an exact interpolator, it creates a smooth surface free of any discontinuities, it is a local method, is spatially adaptive, requires no statistical assumptions, can be applied to small datasets, and is parameter free. However, as with any interpolation method, there will be uncertainty in how well the interpolated field values reflect actual phenomenon values. Using a method based on natural neighbour distance based rates of error calculated for data points via cross-validation, a cross-validation error-distance field can be produced to associate uncertainty with the interpolation. Virtual geography experiments demonstrate that given an appropriate number of data points and spatial-autocorrelation of the phenomenon being interpolated, the natural neighbour interpolation and cross-validation error-distance fields provide reliable estimates of value and error within the convex hull of the data points. While this method does not replace the need for analysts to use sound judgement in their interpolations, for those researchers for whom natural neighbour interpolation is the best interpolation option the method presented provides a way to assess the uncertainty associated with natural neighbour interpolations. 


\section{Discrete natural neighbour interpolation with uncertainty using cross-validation error-distance fields}

${ }_{4}$ Thomas R. Etherington ${ }^{1}$

${ }^{1}$ Manaaki Whenua - Landcare Research, PO Box 69040, Lincoln 7640, New Zealand

6 Corresponding author:

Thomas R. Etherington ${ }^{1}$

8 Email address: EtheringtonT@landcareresearch.co.nz

\section{ABSTRACT}

\section{INTRODUCTION}

Spatially continuous geographic phenomena are often only measured at point locations. Interpolation techniques provide a method to convert such point data into a continuous estimate of the phenomenon, and have become a fundamental computational technique of spatial and geographical analysts with key texts devoting large sections to interpolation methods (Burrough and McDonnell, 1998; O'Sullivan and Unwin, 2010; Slocum et al., 2014).

Natural neighbour (or Sibson) interpolation is an interpolation technique that was first presented by Sibson (1981). The method is based upon a Voronoi (or: Dirichlet, Thiessen) diagram that partitions space to identify those areas that are closest to a set of points (Okabe et al., 2000). Previous authors (Sambridge et al., 1995; Watson, 1999) have noted several useful properties of natural neighbour interpolation: (i) the method is an exact interpolator, in that the original data values are retained at the reference data points; (ii) the method creates a smooth surface free from any discontinuities; (iii) the method is entirely local, as it is based on a minimal subset of data locations that excludes locations that, while close, are more distant than another location in a similar direction; and (iv) the method is spatially adaptive, automatically adapting to local variation in data density or spatial arrangement. To this list I would add: (v) there is no requirement to make statistical assumptions; (vi) the method can be applied to very small datasets as it is not statistically based; and (vii) the method is parameter free, so no input parameters that will affect the success of the interpolation need to be specified.

These properties make natural neighbour interpolation particularly well suited for the interpolation of continuous geographic phenomena from data points that have a highly irregular spatial distribution. 
While the choice of an appropriate interpolation method will always vary on a case by case basis, studies comparing interpolation methodologies with climate and land surface data demonstrate that natural neighbour interpolation is a highly competitive and sometimes optimal technique (Abramov and McEwan, 2004; Bater and Coops, 2009; Hofstra et al., 2008; Lyra et al., 2018; Yilmaz, 2007).

Unfortunately, natural neighbour interpolation can be relatively slow in comparison to other methods (Abramov and McEwan, 2004). The high computational cost arises from the need to insert a new point into the Voronoi diagram for every cell that will make up the interpolation field, and this geometric process becomes increasingly difficult in higher dimensions (Park et al., 2006). This has led to the development of discrete (or digital) natural neighbour interpolation that is significantly quicker than traditional approaches (Park et al., 2006) and has been applied successfully in a geographical context (Keller et al., 2015).

While natural neighbour interpolation has various useful properties, and the discrete form is computationally scalable, there is a great deal of uncertainty associated with any interpolation. Therefore, being able to associate interpolation estimates with some form of uncertainty would be highly desirable. Previous efforts for natural neighbour interpolation have been based upon fitting statistical uncertainty models (Bater and Coops, 2009; Ghosh et al., 2012), but this approach is contrary to natural neighbour interpolation's useful properties (v), (vi), and (vii). Therefore, for those researchers who decide that for their data and objectives natural neighbour interpolation is the best interpolation option, I present an approach to associate the interpolation with a measure of uncertainty that is consistent with all the useful properties of natural neighbour interpolation.

\section{MATERIALS \& METHODS}

\subsection{Discrete natural neighbour interpolation}

In the 2-dimensional planar context that is most relevant to geographical applications, discrete natural neighbour interpolation begins by calculating a discrete Voronoi diagram. First, a raster spatial domain $C$ of cells $c$ is defined such that $c \in C \subset \mathbb{R}^{2}$ and hence each $c$ has coordinate attributes $x, y$ for its centre so all $c_{i}=\left\{x_{i}, y_{i}\right\}$.

The data points are then used to define a set $P$ of $n$ data cells $P=\left\{p_{1}, p_{2}, p_{3}, \ldots, p_{n}\right\}$ where $P \in C$, and each data cell has coordinate attributes for its cell centre $x, y$ and value $z$, so $p_{i}=\left\{x_{i}, y_{i}, z_{i}\right\}$. When multiple data points occur within a raster cell, the resulting data cell has a value $z$ that is the mean of all the data point values.

The discrete Voronoi polygon $V\left(p_{i}\right)$ that contains all the cells that are closest to each data cell can then be defined as

$$
V\left(p_{i}\right)=\left\{c \in C \mid d\left(c \rightarrow p_{i}\right)<d\left(c \rightarrow p_{j}\right) \forall j \neq i\right\}
$$

where $d(c \rightarrow p)$ is the Euclidean distance between the centre of the cells $c$ and $p$. When $c$ is equally distant from more than one $p$ for convenience $c$ is assigned to the $p$ with smallest index. The set of $n$ discrete Voronoi polygons then creates the discrete Voronoi diagram

$$
V(P)=\left\{V\left(p_{1}\right), V\left(p_{2}\right), V\left(p_{3}\right), \ldots, V\left(p_{n}\right)\right\}
$$

that identifies which raster cells are closest to which data cells (Figure 1a) (Okabe et al., 2000). In the process of calculating $V(P)$ another set $D(P \rightarrow C)$ that records the Euclidean distance from the set of data cells $P$ to all raster cells $C$ (Figure 1b) is created. As each data cell $p_{i}$ has an associated value $z_{i}, V(P)$ can be used to interpolate the data cell values across the raster to produce $Z(P)$, which in a geographic information system (GIS) context is equivalent to nearest neighbour interpolation (Burrough and McDonnell, 1998; Tomlin, 1990) (Figure 1c).

To interpolate the data cell values using natural neighbour interpolation, the set of Euclidean distances from an interpolation cell $c_{i}$ to all raster cells $D\left(c_{i} \rightarrow C\right)$ is calculated (Figure 1d). Then the discrete Voronoi polygon for the interpolation cell $V\left(c_{i}\right)$ is defined as

$$
V\left(c_{i}\right)=\left\{c \in C \mid D\left(c_{i} \rightarrow C\right) \leq D(P \rightarrow C)\right\}
$$


(a)

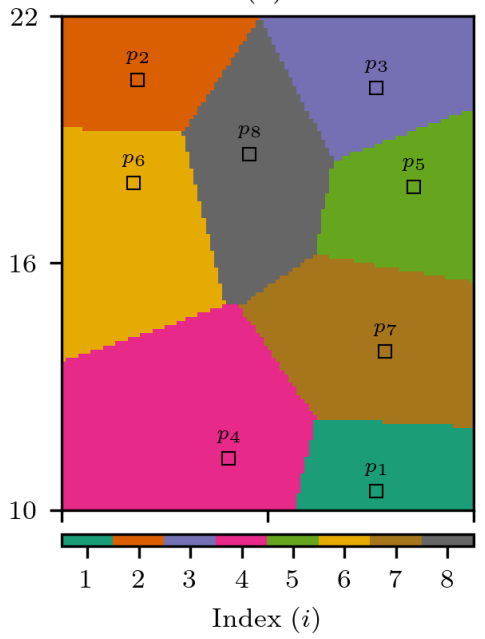

(d)

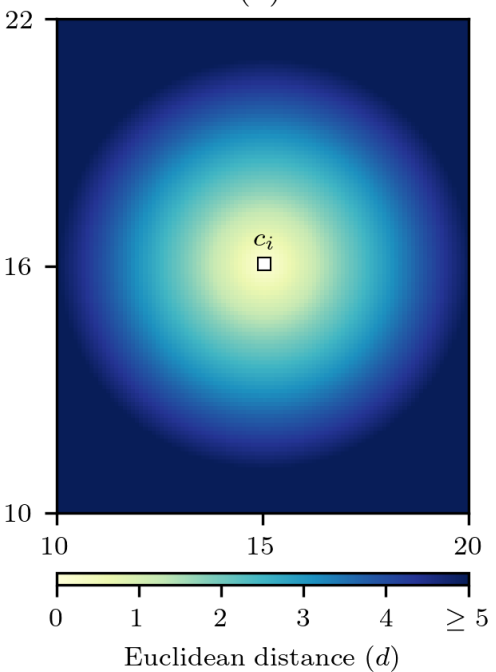

(b)

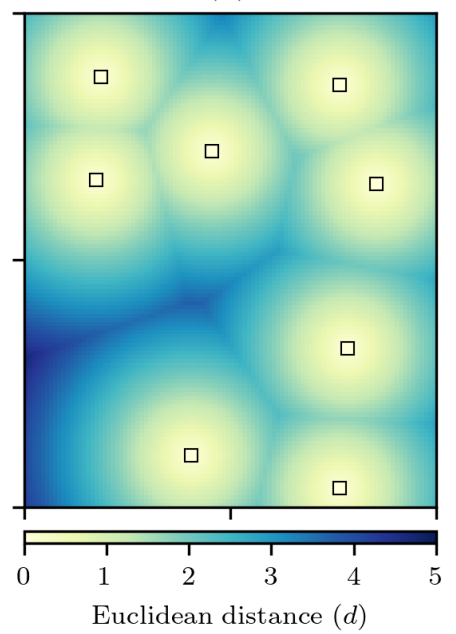

(e)

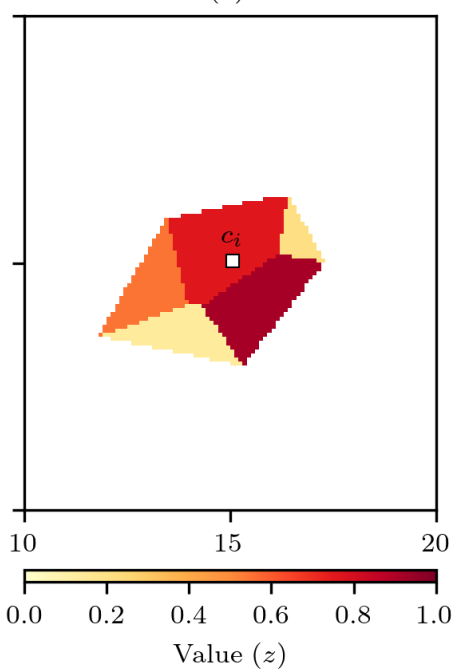

(c)

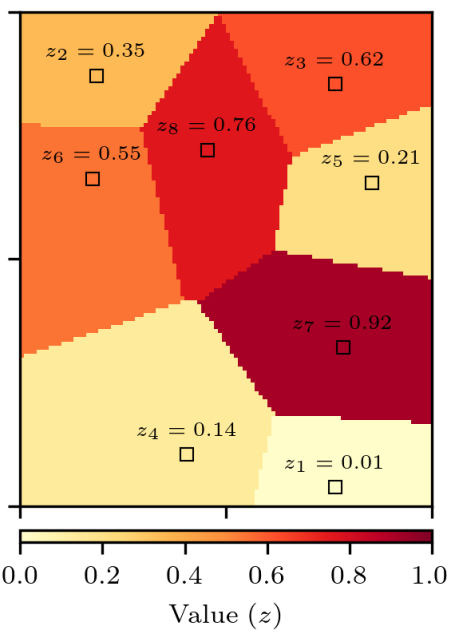

(f)

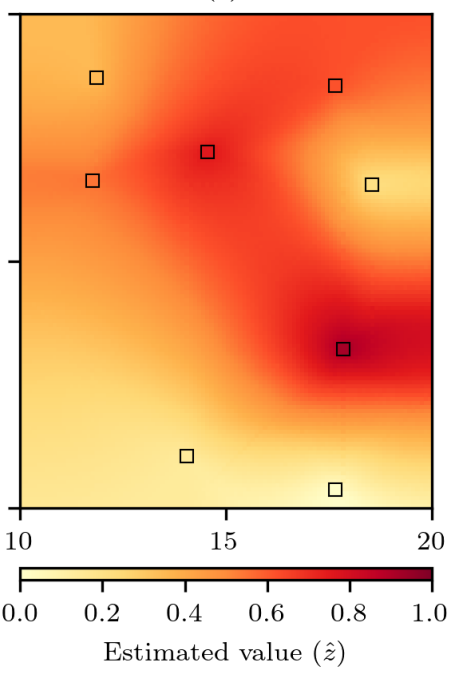

Figure 1. Discrete natural neighbour interpolation. (a) For a set $P$ of $n$ data cells $p$ the discrete Voronoi diagram $V(P)$ defines which raster cells are closest to which data cells and (b) the distance to the closest data cell $D(P \rightarrow C)$. (c) $V(P)$ is used to interpolate the values $z$ of the data cells to produce $Z(P)$. (d) For an interpolation cell $c_{i}$ the distance to all raster cells $C$ is calculated as $D\left(c_{i} \rightarrow C\right)$, and (e) by comparing $D\left(c_{i} \rightarrow C\right)$ to $D(P \rightarrow C)$ identifies $Z\left(c_{i}\right)$ which are those cells of $Z(P)$ that are as close or closer to the $c_{i}$ than any data cell $p$. The mean value of $Z\left(c_{i}\right)$ is the natural neighbour interpolation estimate $\hat{z}$ for $c_{i}$, and by repeating this process for all raster cells (f) the natural neighbour interpolation is produced. 
that is the set of raster cells that are as close or closer to the interpolation cell than any data cell. The set $V\left(c_{i}\right)$ can then be used to find the set of relevant data cell values

$$
Z\left(c_{i}\right)=\left\{c \in Z(P) \mid c \in V\left(c_{i}\right)\right\}
$$

that will form the basis on the interpolation to that cell (Figure 1e). The natural neighbour interpolation estimate $\hat{z}$ is then calculated as

$$
\hat{z}\left(c_{i}\right)=\frac{\sum Z\left(c_{i}\right)}{\sharp Z\left(c_{i}\right)}
$$

where $\sum Z\left(c_{i}\right)$ is the sum of the cell values in $Z\left(c_{i}\right)$ and $\sharp Z\left(c_{i}\right)$ is the number of cells in the set $Z\left(c_{i}\right)$, hence $\hat{z}\left(c_{i}\right)$ is simply the mean of $Z\left(c_{i}\right)$. By calculating $\hat{z}\left(c_{i}\right)$ for all raster cells the natural neighbour interpolation is produced (Figure 1f).

\subsection{Calculating uncertainty} 2.2.1 Cross-validation error

Global error estimation is a traditional approach to measure the uncertainty of geographic models (Zhang and Goodchild, 2002). Given a set of $n$ paired observed $o$ and modelled $m$ values, the absolute error $e_{i}$ for each pair is $e_{i}=\left|m_{i}-o_{i}\right|$, and a global estimate of error using a method such as the mean absolute error (MAE) is calculated as

$$
M A E=\frac{1}{n} \sum_{i=1}^{n} e_{i}
$$

that is simply the mean of all the absolute errors (Willmott and Matsuura, 2005).

However, there is little point in doing this for the data cells of natural neighbour interpolation as given property (i) that it is an exact interpolator the estimated value $\hat{z_{i}}$ for the data cells will always be the same as the actual value $z_{i}$ so the absolute errors will always be zero. Therefore, MAE needs to be applied in conjunction with a cross-validation approach that iteratively withholds each data cell $p_{i}$ from the set of data cells $P$ to produce the set $\left\{P-p_{i}\right\}$, and then uses interpolation to estimate the value $\hat{z}_{i}$ at the withheld data cell $p_{i}$ on the basis of a discrete Voronoi diagram $V\left(\left\{P-p_{i}\right\}\right)$ that is developed without the withheld data cell. The absolute error $e_{i}$ for each data cell $p_{i}$ is then calculated as $e_{i}=\left|\hat{z}_{i}-z_{i}\right|$ and the cross-validation MAE can be calculated using Equation 6.

Even with cross-validation the MAE like all global error estimates, such as the commonly used root-mean-square error (RMSE), are not ideal measures of uncertainty for a spatial interpolation (Zhang and Goodchild, 2002). As non-spatial methods that average errors across space they cannot indicate if errors are consistent across space or if higher errors in one region are balanced out by lower errors in another region. This is a critical limitation of global error estimation methods, as for application purposes it could be very useful to know where the interpolation uncertainty is higher or lower.

\subsubsection{Cross-validation error field}

One way to communicate the spatial uncertainty of geographical information is to map estimates of error (Zhang and Goodchild, 2002). This has been attempted before for natural neighbour interpolation (Bater and Coops, 2009; Ghosh et al., 2012), but as already noted these statistical modelling approaches are contrary to natural neighbour interpolation's useful properties (v), (vi), and (vii).

Another way to map estimates of error that is consistent with the properties of natural neighbour interpolation is the cross-validation error field (Willmott and Matsuura, 2006). This process begins in a similar manner to the cross-validation MAE, but once $e$ has been calculated for each data cell, rather than average the errors using Equation 6 the errors are assumed to be spatially autocorrelated and interpolation is used to interpolate $e$ to estimate an absolute error field $\hat{e}$. This use of localised absolute errors is highly advantageous as it is consistent with property (iii) of natural neighbour interpolation and allows for error estimates to reflect local changes in the spatial-autocorrelation of the phenomenon being interpolated, with lower errors in more autocorrelated areas and higher errors less autocorrelated areas. 
(a)

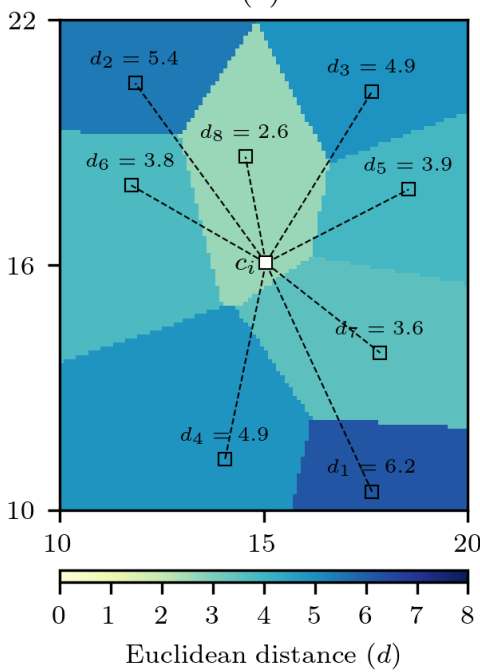

(b)

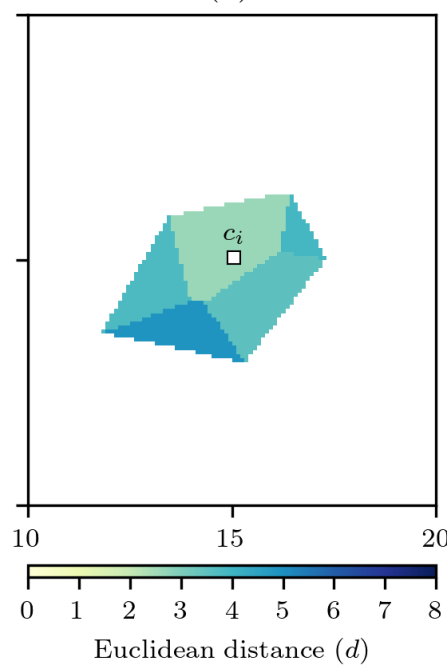

(c)

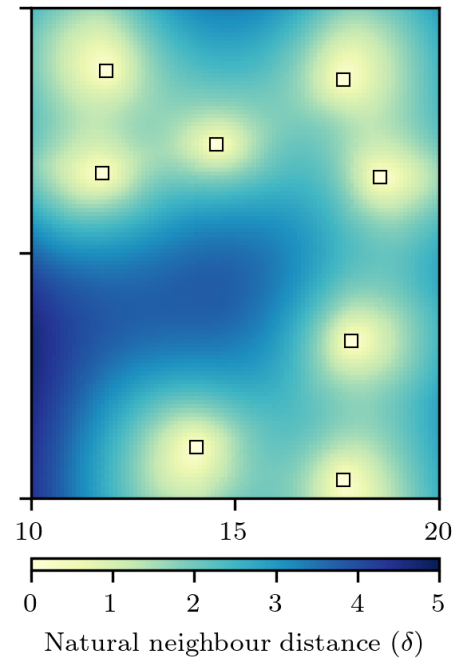

Figure 2. Computation of the natural neighbour distance. (a) For an interpolation raster cell $c_{i}$ the Euclidean distance to all data cells $d_{j}$ is calculated, and the discrete Voronoi diagram $V(P)$ is used to produce $D(P)$ that interpolates the distances by the discrete Voronoi polygons. (b) the cells of $D(P)$ that are closer to $c_{i}$ than any data cell defines the set $D\left(c_{i}\right)$ and the mean value of this set gives the natural neighbour distance $\delta$ for $c_{i}$. (c) When repeated for all raster cells a natural neighbour distance field is produced.

A positive relationship between natural neighbour interpolation absolute errors and the minimum distance to a data cell has been shown (Keller et al., 2015), so this relationship could be used to predict absolute error as a function of distance from the nearest data point. However, minimum distance to a data cell is a simplistic metric that does not account for the number and spatial configuration of the data cells (Keller et al., 2015). In addition, using the minimum distance from data cells $D(P)$ produces a field that has discontinuities along the edges of the discrete Voronoi polygons (Figure 1b) that are contrary to the property (ii) of the natural neighbour interpolation method that creates surfaces free of any discontinuities. Therefore, the natural neighbour distance $\delta$ is presented as a more appropriate measure of distance that incorporates information about the number, spatial distances, and relative positions of the data cells forming the interpolation.

The method to calculate $\delta$ follows a very similar approach to that of calculating the interpolation, and therefore recycles various data structures that are used for the interpolation. For each interpolation cell $c_{i}$ the Euclidean distances to all data cells are calculated $d_{j}=d\left(c_{i} \rightarrow p_{j}\right)$, and then using the Voronoi diagram $V(P)$ these distances are interpolated via nearest neighbour interpolation to produce $D(P)$ that is the distance to the data cells mapped into the discrete Voronoi polygons (Figure 2a).

The set $V\left(c_{i}\right)$ can be used again to find the set of relevant data cell distances 


$$
D\left(c_{i}\right)=\left\{c \in D(P) \mid c \in V\left(c_{i}\right)\right\}
$$

that will form the basis of the interpolation to that cell (Figure 2b). The natural neighbour distance is then calculated as

$$
\delta\left(c_{i}\right)=\frac{\sum D\left(c_{i}\right)}{\sharp D\left(c_{i}\right)}
$$

that is simply the mean value of the distances for the cells in $D\left(c_{i}\right)$. With $\delta$ calculated for all raster cells it becomes evident that unlike minimum distance that contains spatial discontinuities (Figure 1b) the natural neighbour distance creates a smooth surface free of any discontinuities (Figure 2c). Also, the minimum distance is an optimistic measure of distance as it only accounts for the closest data cell, whereas by comparison the distances for $\delta$ are larger as they recognise that the other data cells involved in the interpolation are further away.

\subsubsection{Cross-validation error-distance field}

To incorporate $\delta$ into the estimate of error to produce a cross-validation error-distance field, the first step is still a cross-validation process in which each data cell is iteratively withheld and an estimate of the value of the withheld data cell is made with the remaining $n-1$ data cells. However, the absolute error $e=\left|z_{i}-\hat{z}_{i}\right|$ is now divided by the natural neighbour distance $\delta$ to calculate a rate of error $r$ for each data cell

$$
r_{i}=\frac{\left|z_{i}-\hat{z}_{i}\right|}{\delta_{i}}
$$

with these rates of error stored so that each data cell becomes $p_{i}=\left\{x_{i}, y_{i}, z_{i}, r_{i}\right\}$. Then when conducting the natural neighbour interpolation, while estimating the value $\hat{z}$ an estimate of the rate of error $\hat{r}$ can be simultaneously produced (Figure $3 \mathrm{a}$ ) and used to produce an error estimate

$$
\hat{e}_{i}=\hat{r}_{i} \times \delta_{i}
$$

that when estimated for all cells produces a cross-validation error-distance field (Figure 3b). The cross-validation error-distance field clearly captures information from the rate of error field (Figure 3a) and the natural neighbour distance field (Figure 2c) with lower error estimates in areas that have either low rates of error or natural neighbour distances, and higher error estimates in areas that have higher rates of error and/or natural neighbour distances. Therefore, the cross-validation error-distance field captures uncertainty information relating to local variation in both the autocorrelation of the underlying phenomenon field being interpolated and the spatial distribution of the data cells providing data for the interpolation.

\subsection{Virtual geography experiments}

The discrete natural neighbour interpolation and cross-validation error-distance field algorithms described here were implemented using a Python computational framework (Pérez et al., 2011) using the NumPy (van der Walt et al., 2011), SciPy (Virtanen et al., 2020), and Matplotlib (Hunter, 2007) packages. Having proposed a new method, it is sensible to provide an evaluation of how performance varies under different conditions. However, in doing so it is important to remember that interpolation errors result not only from the efficacy of the interpolation method, but also from distribution of data points and the real (but unknown) distribution of the phenomenon field being interpolated (Willmott and Matsuura, 2006) that will be unique to each study. Also, what constitutes an acceptable level of interpolation error will also vary between studies. Therefore, the objective here is try and identify simple trends in performance to verify the methods work as would be expected and to provide some basic information that will help an analyst to make a more detailed assessment of whether interpolation is feasible or not.

To evaluate the effectiveness of the proposed interpolation methods, a series of in silico virtual geography experiments were conducted. Virtual geographies are a very useful approach for methodological 
(a)

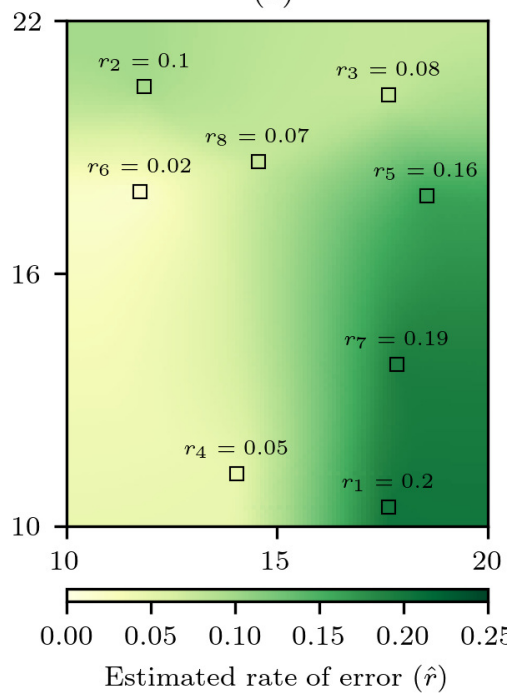

(b)

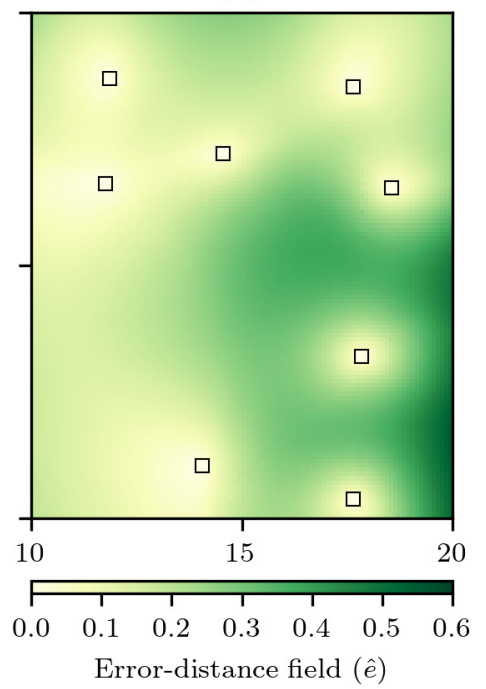

Figure 3. Computation of the cross-validation error-distance field. (a) The rate of absolute error for each data cell $r_{i}$ calculated through cross-validation, and then an estimated rate of absolute error field $\hat{r}$ is produced by natural neighbour interpolation of $r$. (b) The cross-validation error-distance field $\hat{e}$ that is the product of $\hat{r}$ and the natural neighbour distance $\delta$ for each interpolation cell.

evaluation as the conditions can be tightly controlled and explored fully. Virtual geographic phenomena fields for grids of $100 \times 120$ cells were created using the NLMpy package (Etherington et al., 2015) implementation of the mid-point displacement fractal algorithm that produces fields representing natural phenomena such as land surfaces (Fournier et al., 1982). The spatial-autocorrelation of the values produced by the mid-point displacement method can be controlled by varying the $h$ parameter to produce fields with spatial-autocorrelation that varies from low to high (Figure 4).

The underlying premise of the experiments was that with random sampling of a virtual geographic phenomenon with actual values $z$ (Figure 5a), natural neighbour interpolation can be used to produce estimated values $\hat{z}$ (Figure $5 b$ ). The absolute difference between the actual values and the estimated values is the value error $e(\hat{z})=|\hat{z}-z|$ (Figure 5c) that will indicate how well the natural neighbour interpolation method works. The value error is also estimated by the cross-validation error-distance field $\hat{e}$ (Figure $5 \mathrm{~d})$, and the absolute difference between the value error $e(\hat{z})$ and the estimated error $\hat{e}$ is the error of errors $e(\hat{e})=|\hat{e}-e(\hat{z})|$ that indicates how well the proposed cross-validation error-distance field performs (Figure 5e).

To summarise the performance of both natural neighbour interpolation and the cross-validation errordistance field, the MAE (Equation 6) was calculated for the cells inside and outside of the convex hull of the sampling points for both $e(\hat{z})$ (Figure $5 \mathrm{c}$ ) and $e(\hat{e})$ (Figure 5e). The MAE was chosen as the error statistic as it expresses error in the same units as the variable of interest and is insensitive to the number of cells in the sample (Willmott and Matsuura, 2006), which was important here as the convex hull area would vary as a result of the random sampling.

When the spatial-autocorrelation and number of sample points is reduced we would expect a reduction in performance of both the natural neighbour interpolation and the cross-validation error-distance field (Figure 5a-e versus Figure 5f-j). Therefore, to examine how the natural neighbour methods performed under varying conditions 500 experiments were conducted in which $h$ randomly varied uniformly between 0.0 to 2.0 and $n$ randomly varied uniformly between 10 to 100 . The cross-validation MAE was also calculated for each experiment to assess if the cross-validation MAE could be used as an indicator of expected interpolation performance. 
(a)

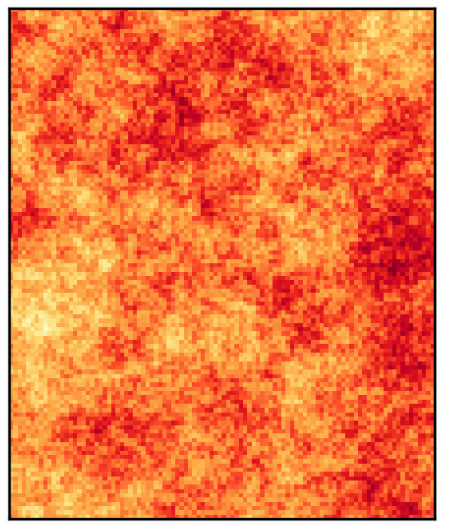

(b)

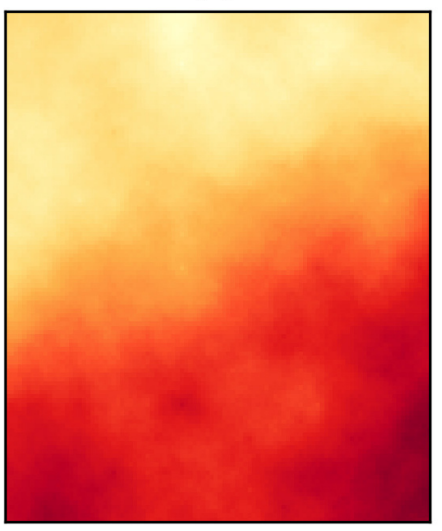

(c)

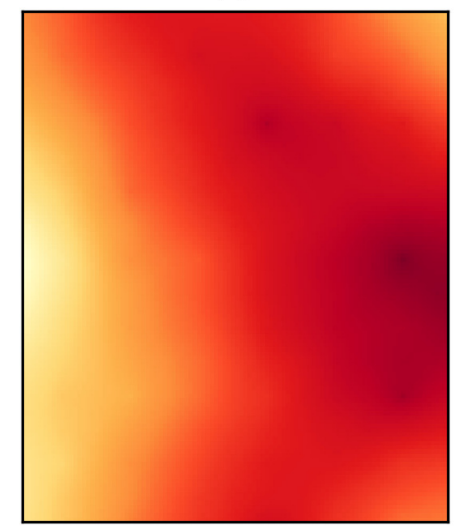

$\begin{array}{ll}1.0 & 0.0\end{array}$
1.00 .0

0.0

0.5

Value $(z)$

Figure 4. Examples of virtual geographic phenomena fields created by the mid-point displacement fractal algorithm. The spatial-autocorrelation varies from low to high and is controlled by the $h$ parameter that in these examples has been set to (a) $h=0$, (b) $h=1$, and (c) $h=2$.

\section{RESULTS}

The results from the virtual geography experiments demonstrate that, as would be expected for the cells within the convex hull of the sampling points, the MAE of the value errors $e(\hat{z})$ from the natural neighbour interpolation (Figure 6a) and error of errors $e(\hat{e})$ from the cross-validation error-distance field (Figure 6b) reduced as the number of data points $n$ and the spatial-autocorrelation $h$ of the underlying virtual phenomena fields increased. The effect of $h$ was more important, as when $h$ was low or high $n$ did not have much effect on the performance. The importance of $h$ is to be expected as all interpolation methods work on the assumption that the phenomenon being interpolated has sufficient levels of spatial-autocorrelation.

There was also a very strong correlation between $e(\hat{z})$ and $e(\hat{e})$ (Figure 6c) and this similarity of behaviour under different conditions indicates that the cross-validation error-distance field meets the objective of providing a measure of uncertainty that is consistent with all the useful properties of natural neighbour interpolation.

While the results of the virtual geography experiments (Figure $6 \mathrm{a}$ and $\mathrm{b}$ ) indicate that lower average errors can be expected when $n \gtrsim 20$ and $h \gtrsim 1.0$ (Figure $4 \mathrm{~b}$ ) such criteria cannot be easily applied by an analyst as while $n$ is known $h$ is unknown and in many situations will be hard to guess. Fortunately, while the cross-validation MAE that can always be calculated by an analyst is generally slightly higher than the $e(\hat{z})$ there is still a strong correlation between the two variables (Figure 6d), and this correlation is extremely useful as it indicates to an analyst the likely levels of $e(\hat{z})$ and therefore $e(\hat{e})$ too.

A comparison of $e(\hat{z})$ and $e(\hat{e})$ inside and outside of the convex hull around the sampling points clearly shows that while the performance follows a similar trend $e(\hat{z})$ and $e(\hat{e})$ can be expected to be higher outside of the convex hull (Figure 6e-f).

\section{DISCUSSION}

The virtual geography experiments indicate that under suitable conditions the natural neighbour interpolation field and the cross-validation error-distance field should provide useful estimates of a geographic phenomenon field with associated uncertainty. The fact that the cross-validation error-distance field reflects localised changes in the spatial distribution of both the underlying phenomenon and the point data is particularly useful, and contrasts with other spatial interpolation uncertainty methods such as MAE and RMSE that estimate error using a global approach.

The virtual geography experiments demonstrated that the performance of natural neighbour interpolation will be lower outside of the convex hull around the data points, as is expected (Watson, 1999) although this is also likely to be true of all spatial interpolation techniques as beyond the convex hull 
(a)

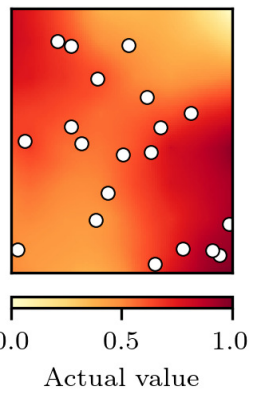

(f)

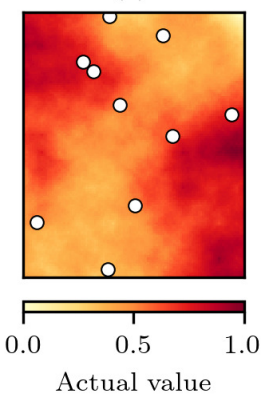

(b)

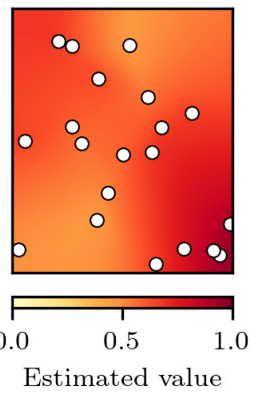

(g)

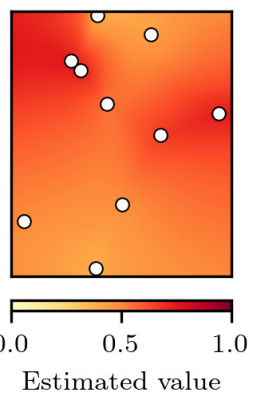

(c)

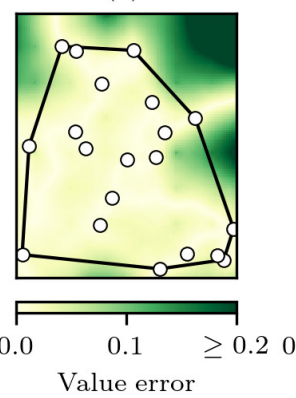

(h)

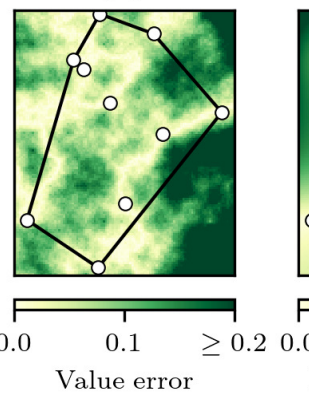

(d)

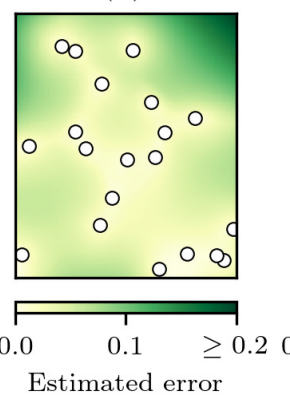

(i)

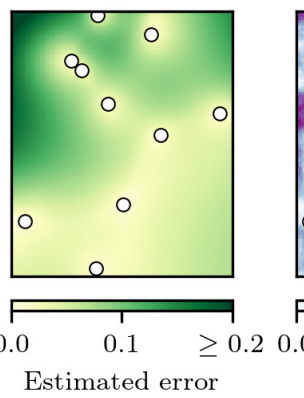

(e)

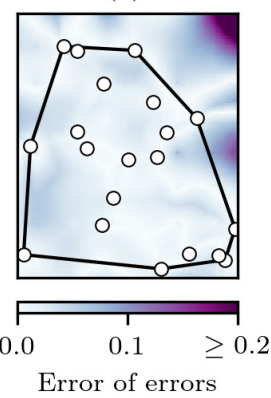

(j)

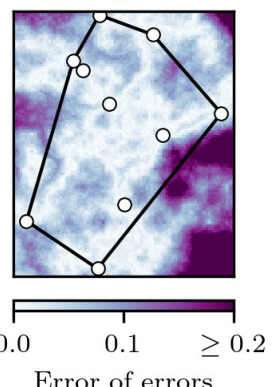

Figure 5. The natural neighbour interpolation virtual geography experimental process. (a) A virtual geography phenomenon field $z$ with spatial-autocorrelation of $h=2$ and $n=20$ random sampling points, (b) the resulting natural neighbour interpolation $\hat{z}$ from the sampling points, and (c) value error $e(\hat{z})=|\hat{z}-z|$. (d) The cross-validation error-distance field estimated error $\hat{e}$ that is also produced during interpolation is then compared to the value error $e(\hat{z})$ to produce (e) the error of errors $e(\hat{e})=|\hat{e}-e(\hat{z})|$. Interpolation performance as a function of $e(\hat{z})$ and $e(\hat{e})$ was summarised for cells within and outside the convex hull of the sampling points. The same experimental process in $(a-e)$ is replicated in $(f-j)$ for a virtual geography phenomenon field with spatial-autocorrelation of $h=1$ and $n=10$ random sampling points, demonstrating a reduction in interpolation performance at lower levels of spatial-autocorrelation and sampling.

interpolation becomes extrapolation. However, we do not suggest that interpolation should be restricted to within the convex hull as there may be occasions where the area of interest may occur slightly outside the convex hull. For example, when interpolating rainfall data from weather stations that are usually sited in settlements, there are likely to be areas of coastline along peninsulas and headlands that will not fall within a convex hull around the weather stations (Lyra et al., 2018). Therefore, it is logistically useful that discrete natural neighbour interpolation can produce estimated values beyond the convex hull of the available data points. What is helpful in this context is that the cross-validation error-distance field incorporates information on distance from data points, therefore as interpolations move further beyond the convex hull the error-field should increase to help to guard against erroneous estimates.

However, the responsibility of appropriate use of natural neighbour interpolation still belongs with the spatial analyst who must make decisions about whether interpolation is useful based on their knowledge of: the expected spatial-autocorrelation of the phenomenon being interpolated, the number and distribution of data points, the location of the areas for which interpolations are required, and the magnitude of the estimated errors in relation to the magnitude of the value estimates. And of course, the cross-validation error-distance field only captures uncertainty in the interpolation itself and does not incorporate any uncertainty that may arise from the data itself. While I have argued against the use of the cross-validation MAE as a measure of uncertainty, I would recommend that analysts continue to calculate the crossvalidation MAE given its strong correlation with the performance of the natural neighbour interpolation, and therefore the performance of the cross-validation error-distance field too. Analysts can then use the cross-validation MAE as a helpful guide when deciding if interpolation is advisable or not. When doing 
so it is important to remember that as the cross-validation MAE is based on the use of $n-1$ data cells, the error estimates may be slightly higher than the real errors that would be based on all $n$ data that is ultimately used in the interpolation (Willmott and Matsuura, 2006). Therefore, the cross-validation MAE should be seen as a slightly conservative indication of likely interpolation performance.

\section{CONCLUSION}

For those researchers for whom natural neighbour interpolation is the best interpolation option, the cross-validation error-distance field method presented provides a way to assess the uncertainty associated with natural neighbour interpolations that is consistent with the useful properties of natural neighbour interpolation. While the cross-validation error-distance method has been described here in the context of discrete natural neighbour interpolation, there is no reason why this same approach could not be applied to geometric natural neighbour interpolation as well. Discrete natural neighbour interpolation has been implemented here in two-dimensional space for ease of visualisation, but the method will generalise to higher dimensions (Park et al., 2006) and in principle I cannot see any reason why the uncertainty method presented could not also be applied in higher dimensions by those who wish to do so. The approach could easily be adapted to other interpolation methods, as all that is required is a measure of weighted distances to the data points creating the interpolation. Given the promise of the algorithm, and to encourage its use and development, the Python code used to generate the examples presented is freely available under the permissive MIT License as supplementary material.

\section{REFERENCES}

Abramov, O. and McEwan, A. (2004). An evaluation of interpolation methods for Mars Orbiter Laser Altimeter (MOLA) data. International Journal of Remote Sensing, 25(3):669-676.

Bater, C. W. and Coops, N. C. (2009). Evaluating error associated with lidar-derived DEM interpolation. Computers \& Geosciences, 35(2):289-300.

Burrough, P. A. and McDonnell, R. A. (1998). Principles of Geographical Information Systems. Oxford University Press, Oxford.

Etherington, T. R., Holland, E. P., and O'Sullivan, D. (2015). NLMpy: a Python software package for the creation of neutral landscape models within a general numerical framework. Methods in Ecology and Evolution, 6(2):164-168.

Fournier, A., Fussell, D., and Carpenter, L. (1982). Computer rendering of stochastic models. Communications of the ACM, 25(6):371-384.

Ghosh, S., Gelfrand, A. E., and Mølhave, T. (2012). Attaching uncertainty to deterministic spatial interpolations. Statistical Methodology, 9(1-2):251-264.

Hofstra, N., Haylock, M., New, M., Jones, P., and Frei, C. (2008). Comparison of six methods for the interpolation of daily, European climate data. Journal of Geophysical Research, 113(D21):D21110.

Hunter, J. D. (2007). Matplotlib: a 2D graphics environment. Computing in Science \& Engineering, 9(3):90-95.

Keller, V. D. J., Tanguy, M., Prosdocimi, I., Terry, J. A., Hitt, O., Cole, S. J., Fry, M., Morris, D. G., and Dixon, H. (2015). CEH-GEAR: $1 \mathrm{~km}$ resolution daily and monthly areal rainfall estimates for the UK for hydrological and other applications. Earth System Science Data, 7(1):143-155.

Lyra, G. B., Correia, T. P., de Oliveira-Júnior, J. F., and Zeri, M. (2018). Evaluation of methods of spatial interpolation for monthly rainfall data over the state of Rio de Janeiro, Brazil. Theoretical and Applied Climatology, 134(3):955-965.

Okabe, A., Boots, B., Sugihara, K., and Chiu, S. N. (2000). Spatial Tessellations: concepts and applications of Voronoi diagrams. John Wiley \& Sons, Chichester, 2nd edition.

O'Sullivan, D. and Unwin, D. J. (2010). Geographic Information Analysis. John Wiley \& Sons, Hoboken, 2nd edition.

Park, S. W., Linsen, L., Kreylos, O., Owens, J. D., and Hamann, B. (2006). Discrete Sibson interpolation. IEEE Transactions on Visualization and Computer Graphics, 12(2):243-253.

Pérez, F., Granger, B. E., and Hunter, J. D. (2011). Python: an ecosystem for scientific computing. Computing in Science \& Engineering, 13(2):13-21.

Sambridge, M., Braun, J., and McQueen, H. (1995). Geophysical parametrization and interpolation of irregular data using natural neighbours. Geophysical Journal International, 122(3):837-857. 
Sibson, R. (1981). A brief description of natural neighbour interpolation, pages 21-36. Wiley, Chichester. Slocum, T. A., McMaster, R. B., Kessler, F. C., and Howard, H. H. (2014). Thematic Cartography and Geovisualization. Pearson Education Limited, Harlow.

Tobler, W. (1970). A computer movie simulating urban growth in the Detroit region. Economic Geography, 46(2):234-240.

Tomlin, C. D. (1990). Geographic Information Systems and Cartographic Modeling. Prentice-Hall, Englewood Cliffs.

van der Walt, S., Colbert, S. C., and Varoquaux, G. (2011). The NumPy array: a structure for efficient numerical computation. Computing in Science \& Engineering, 13(2):22-30.

Virtanen, P., Gommers, R., Oliphant, T. E., Haberland, M., Reddy, T., Cournapeau, D., Burovski, E., Peterson, P., Weckesser, W., Bright, J., van der Walt, S. J., Brett, M., Wilson, J., Millman, K. J., Mayorov, N., Nelson, A. R. J., Jones, E., Kern, R., Larson, E., Carey, C. J., Polat, I., Feng, Y., Moore, E. W., VanderPlas, J., Laxalde, D., Perktold, J., Cimrman, R., Henriksen, I., Quintero, E. A., Harris, C. R., Archibald, A. M., Ribeiro, A. H., Pedregosa, F., van Mulbregt, P., Vijaykumar, A., Bardelli, A. P., Rothberg, A., Hilboll, A., Kloeckner, A., Scopatz, A., Lee, A., Rokem, A., Woods, C. N., Fulton, C., Masson, C., Häggström, C., Fitzgerald, C., Nicholson, D. A., Hagen, D. R., Pasechnik, D. V., Olivetti, E., Martin, E., Wieser, E., Silva, F., Lenders, F., Wilhelm, F., Young, G., Price, G. A., Ingold, G.-L., Allen, G. E., Lee, G. R., Audren, H., Probst, I., Dietrich, J. P., Silterra, J., Webber, J. T., Slavič, J., Nothman, J., Buchner, J., Kulick, J., Schönberger, J. L., de Miranda Cardoso, J. V., Reimer, J., Harrington, J., Rodríguez, J. L. C., Nunez-Iglesias, J., Kuczynski, J., Tritz, K., Thoma, M., Newville, M., Kümmerer, M., Bolingbroke, M., Tartre, M., Pak, M., Smith, N. J., Nowaczyk, N., Shebanov, N., Pavlyk, O., Brodtkorb, P. A., Lee, P., McGibbon, R. T., Feldbauer, R., Lewis, S., Tygier, S., Sievert, S., Vigna, S., Peterson, S., More, S., Pudlik, T., Oshima, T., et al. (2020). SciPy 1.0: fundamental algorithms for scientific computing in Python. Nature Methods, 17(3):261-272.

Watson, D. (1999). The natural neighbor series manuals and source codes. Computers \& Geosciences, 25(4):463-466.

Willmott, C. J. and Matsuura, K. (2005). Advantages of the mean absolute error (MAE) over the root mean square error (RMSE) in assessing average model performance. Climate Research, 30(1):79-82.

Willmott, C. J. and Matsuura, K. (2006). On the use of dimensioned measures of error to evaluate the performance of spatial interpolators. International Journal of Geographical Information Science, 20(1):89-102.

Yilmaz, H. M. (2007). The effect of interpolation methods in surface definition: an experimental study. Earth Surface Processes and Landforms, 32(9):1346-1361.

Zhang, J. and Goodchild, M. (2002). Uncertainty in Geographical Information. Taylor \& Francis, London. 
(a)

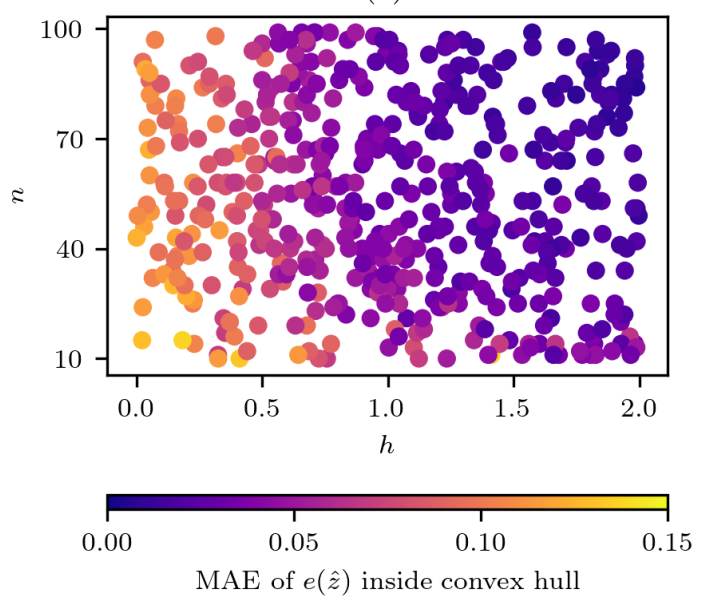

(c)

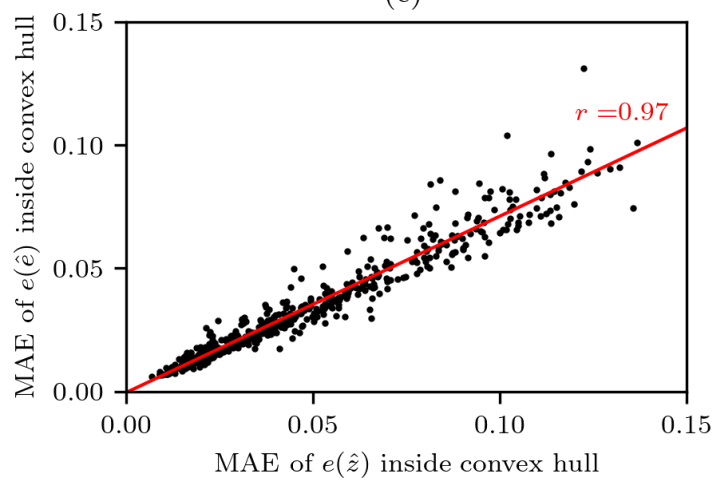

(e)

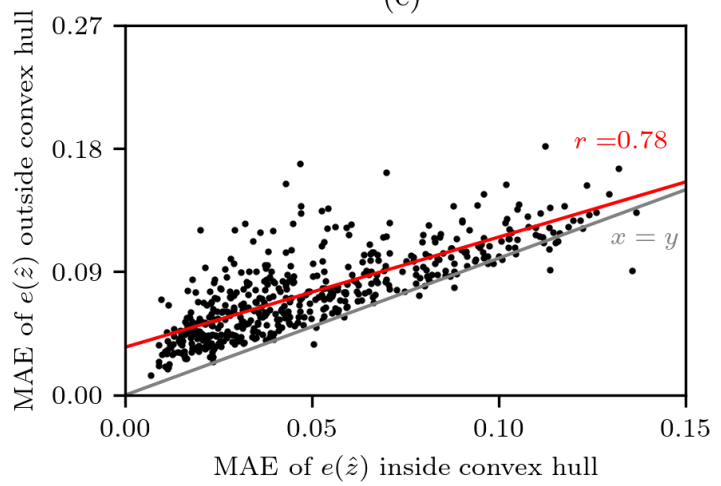

(b)

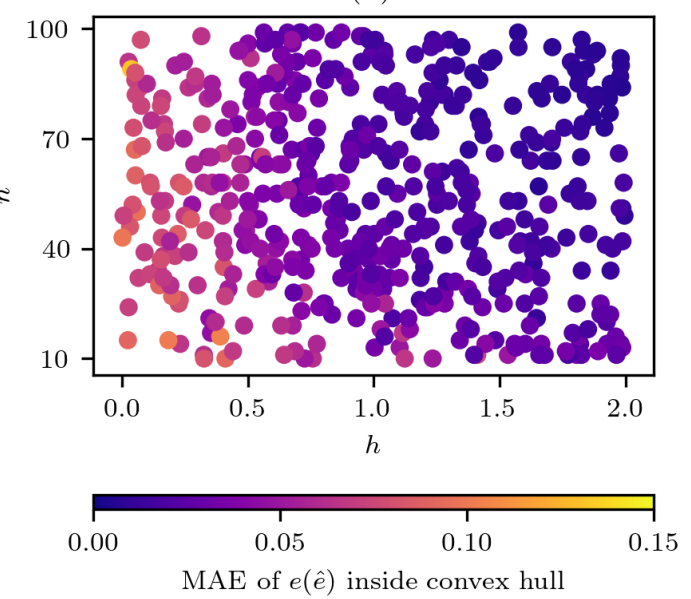

(d)

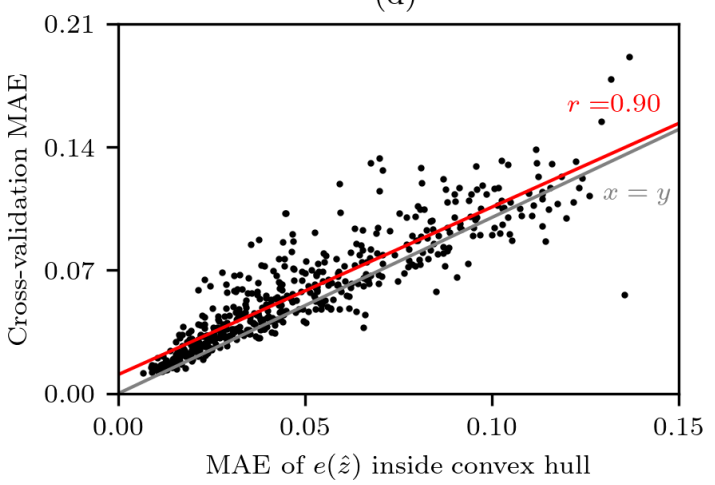

(f)

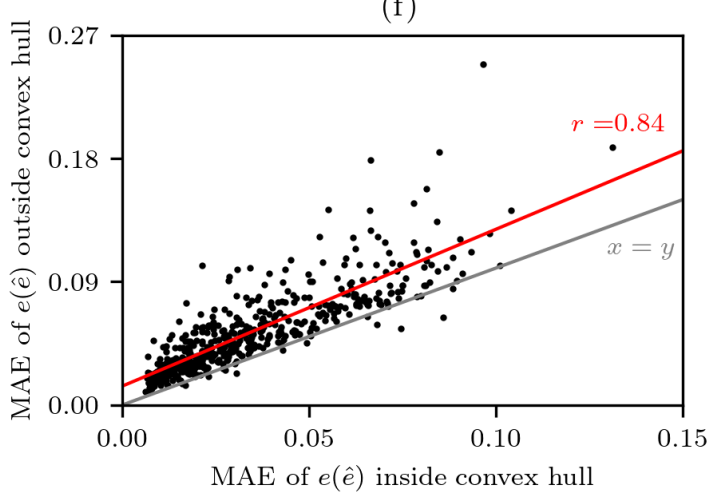

Figure 6. Performance of natural neighbour interpolation and cross-validation error-distance fields from 500 virtual geography experiments. The mean absolute error (MAE) of cells within the convex hull around sampling points for different experimental combinations of the number $n$ of random sampling points and the spatial-autocorrelation $h$ of virtual phenomena fields for (a) the value errors $e(\hat{z})$ from the natural neighbour interpolations and (b) the error of errors $e(\hat{e})$ from the cross-validation error-distance fields that (c) were highly correlated. (d) Comparison of $e(\hat{z})$ and the cross-validation MAE derived from the sampling points. Comparison of interpolation performance inside and outside the convex hull around the sampling points for (e) $e(\hat{z})$ and (f) $e(\hat{e})$. 